\title{
Anti-Nutritional Compounds, Digestibility, Growth Responses, and Body Compositions of Clarias Gariepinus (Burchelle 1882) Fingerlings Fed Differently Processed Locust Bean (Parkia Biglobosa) Meal
}

\author{
Orire $\mathrm{AM}^{*}$ and Ogbonna $\mathrm{CK}$ \\ Department of Water Resources, University of Federal Technology, Nigeria
}

Submission: September 10, 2017; Published: December 12,2017

*Corresponding author: Orire AM, Department of Water Resources, Aquaculture and Fisheries Technology, University of Federal Technology, Minna, P.M.B. 65, Nigeria, Email: abdul.orire@futminna.edu.ng

\begin{abstract}
One of the challenges facing aquaculture industry is high cost of fishmeal. Thus, the need for researches into alternative protein sources especially, from non-competitive plant protein sources becomes imperative. Locust bean (Parkia biglobosa) is a plant protein source whose nutrients usability was experimented upon in the African catfish fingerlings (Clarias gariepinus) of mean weight (3.46 $\pm 0.01 \mathrm{~g}$ ). Five hundred and eight five (585) fishes were randomly distributed in 20 fishes in triplicate. They were fed 13 experimental diets at 50\% crude protein containing differently processed locust bean meal (raw, boiled, fermented and toasted) at four inclusion levels ( $0 \%, 5 \%, 10 \%$ and $15 \%)$. They were fed at $3 \%$ body weight that was adjusted fortnightly. The results obtained indicated significant differences for the treatments.

The fermented and toasted locust bean meal performed best at 5\% inclusion level with mean weigh gains (5.84 \& 5.47gs), Specific Growth Rare (2.00 \& 1.67), Feed Conversion Rate (1.02 \& 1.13), Protein Efficiency Ratio (2.16 \& 1.84) Apparent Net Protein Utilization (178.21 \& $168.0)$ while the raw and boiled locust bean meal based diets had their best impact at $15 \%$ inclusion level with mean weight gain $(5.13$ \& $5.01 \mathrm{gs})$, specific growth rate ( 2.00 \& 1.62\%/Days), Feed Conversion Rates (1.24 \& 1.10) while, the raw locust bean diet had the best Protein Efficiency Ratio (1.99) and Apparent Net Protein utilization value of 124.63. The biological values were through reflections of the Parkia biglobosa's digestibility and body compositions of the experimental fish. The study recommended that, Parkia biglobosa can replace fishmeal as a viable protein source for enhancement of growth, body composition of Clarias gariepinus without any adverse effect at the appropriate replacement levels so discovered.
\end{abstract}

Keywords: Alternative protein source; Locust bean meal; Fish meal; Anti-nutritional compound; Digestibility

\section{Introduction}

Fish is one of the most versatile commodities and that can be used in different ways and product forms. It is distributed as live, fresh, chilled, heat treated, dried, smoked, salted, boiled, fried, powdered or canned, and due to its several uses, the demand for fish is always on the rise [1]. The declining stocks of fish from capture fishery, competition for feed in animal husbandry, high cost and scarcity of fish have prompted investigation into the ways of replacing or substituting fish meal with less expensive feed stuff in a developing country like Nigeria [2]. The superiority of fishmeal in growth performance is due mainly to its nutritional composition and its amino acid profile. However, it has been established in all fish diets, irrespective of species, that, protein is the most important dietary nutrient and fish meal remains one of the major sources of dietary protein $[3,4]$.

Rapid development of fish farming calls for greatly stepped up production of locally produced quality fish feed since fish feed accounts for at least forty-sixty percent (40-60\%) of management cost in Aquaculture [5]. Catfish is considered as an affordable and acceptable substitute for white fish. In Vietnam, it is regarded as "princess". Catfish grow fast and are easily accepted in all communities in Nigeria. They equally attract a good market price during harvest. The fish have mostly been used" to control over breeding in earthen pond: in mixed-sex tilapia culture. Besides, smoked catfish are also in high demand because as they 
can be stored for longer periods while retaining its nutritional quality [6]. In the tropic, locust bean seed is available and less expensive. It is commonly consumed in its fermented forms [7].

Locust bean seed when fermented (Dawadawa) is rich in protein, Lipids and Vitamin B2, and lysine [8] which is lacking in other leguminous plant and important for fish growth. Locust bean seed have extensive uses domestically as condiments, flavor intensifier for soups and stews and it also adds protein to diet of poor [9]. However, typical of leguminous seeds, locust bean seed have been reported of anti-nutritional compounds which may limit its availability for fish growth [10]. In view of the high cost of fish meal and its dwindling availability, there is the need to source for alternate plant protein source that is not in direct competition with man's need for fish feed production. This research seeks to investigate the effect of processing on nutrient availability of locust bean seed on the growth of Clarias gariepinus.

\section{Materials and Methods}

\section{Experimental site}

The research was carried out in the Research and Teaching Laboratory of Department of Water Resources, Aquaculture and Fisheries Technology Federal University of Technology, Minna,
Niger State, Nigeria. The experimental design was Randomized Complete Block Design (RCBD) where a total number of five hundred and eighty five (585) catfish (Clarias gariepinus) fingerlings $(3.14 \pm 0.52 \mathrm{~g})$ were obtained from a hatchery farm in New Bussa, Niger State and transported to the laboratory where they were acclimatized for two weeks. 15 fishes were randomly stocked in triplicates of 39 tanks of dimensions of $30 \times 60 \times 30 \mathrm{~cm}$ and filled up with 20 litres of bore whole water in a recirculation water system. Fishes were $3 \%$ body weight daily and was adjusted fortnightly (5\%, $7 \%$ and $9 \%$ ) to meet body requirement, dead fishes were picked daily, feacal matters and uneaten feeds were siphoned with daily recycling of water for optimization of dissolved oxygen concentration. The water quality parameters were measured using clinical thermometer for temperature and dissolve oxygen was determined according to Wrinker's method. Hydrogen ion concentration $(\mathrm{pH})$ was measured using an EL 7045/46 at room temperature while conductivity was monitored with conductivity meter $(\mu \mathrm{m})$ $[11,12]$ the water quality parameters measured is in (Table 1 ). The feeding trial lasted for eight (8) weeks. The fishes were fed experimental diets as formulated to approximately 50\% crude protein which contained four levels of inclusion of locust bean seeds $(0 \%, 5 \%, 10 \%$ and $15 \%)$ with four processing methods (raw, boiled, fermented and toasted).

Table 1: Proximate compositions of feed stuffs.

\begin{tabular}{|c|c|c|c|c|c|c|c|}
\hline Samples & \%Crude Protein & \%Lipid & \%Ash & \%Crude Fiber & \%Moisture Content & \%NFE & \%DM \\
\hline Raw & 31.84 & 9.98 & 5.87 & 7.63 & 10.53 & 34.15 & 89.47 \\
\hline Boiled & 34.00 & 18.65 & 4.88 & 6.64 & 7.23 & 28.60 & 92.77 \\
\hline Fermented & 38.46 & 21.8 & 5.12 & 7.22 & 11.10 & 16.3 & 88.94 \\
\hline Toasted & 38.27 & 18.36 & 11.35 & 12.05 & 3.37 & 16.6 & 96.63 \\
\hline Maize (Yellow) & 12.60 & 5.13 & 1.89 & 1.60 & $12.40-$ & 66.38 & 87.6 \\
\hline Fish Meal & 65.00 & 10.82 & 6.65 & 1.35 & 14.4 & 1.78 & 85.6 \\
\hline
\end{tabular}

\section{Processing of locust bean meal}

Processing of raw locust bean meal: $300 \mathrm{~g}$ of the seeds was weighed for sorting from dirts, they were dehulled and oven dried to attain constant moisture content. The seeds were then milled in attrition milling machine and sieved through $0.4 \mathrm{~mm}$ wire mesh. The raw African locust bean flour was packed in plastic container sealed with aluminium foil and stored in a freezer at $0{ }^{\circ} \mathrm{C}$ prior to analysis. This preparation was in according to the method of [7].

Boiled locust bean meal: The method of Fakunle JO [13] was used. $300 \mathrm{gm}$ of the seeds were measured, sorted, and made to boiled at $100{ }^{\circ} \mathrm{C}$ for $6 \mathrm{hrs}$. The boiled seeds were cooled under shade and air dried. This was then ground and packed for storage in a freezer at $0{ }^{\circ} \mathrm{C}$.

Fermentation locust bean meal: It was done according to the method of Yusuf \& Rahji [14]. 300g of African locust bean seeds were boiled in water for $12 \mathrm{hrs}$, it was allowed to cool, the seed coats were removed by pressure between palms and washed. The cotyledons were reboiled in water for $1-2 \mathrm{hrs}$, and were drained through raffia sieve. The seeds were hence, spread on wide calabash tray, and then wrapped with jute bags and set to ferment which took place after $36 \mathrm{hrs}$.

Toasted locust bean meal: The toasting processing was done according to the method of Nwachukwu et al. [15]. The locust bean seed were thoroughly sorted to remove all extraneous materials. The clean seeds were toasted for about 15 minutes at 100 at $0{ }^{\circ} \mathrm{C}$ when seed coat starts popping, and then dehulled which was followed by winnowing, milling and sieving. The flour was then packed and stored in a freezer at $0{ }^{\circ} \mathrm{C}$ temperature.

\section{Chemical Analysis}

Proximate chemical analysis was carried out for feedstuffs, formulated diets and carcass for evaluation of crude protein, crude fiber, lipid, and ash and moisture contents using the Macro Kjeldahl method as described by Association of Official Analytical Chemists (2000). 


\section{Determination of anti-nutritional factors}

Determination of tanin content: The level of Tanin in the seed and the processed seeds were determined by the Folin Denis spectrophotometer method of Pearson [16]. It was done by measuring $1.0 \mathrm{~g}$ of sample, dispersed in distilled water and agitated. This was left to stand for 30 minutes at room temperature, with intermittent shaken at an interval of 5 minutes. After 30 minutes, it was centrifuged and the extraction was collected. $2.5 \mathrm{ml}$ of the supernatant (extracted) was dispersed into a $50 \mathrm{ml}$ volumetric flask. A $1.0 \mathrm{ml}$ Folin Denis reagent was measured into each flask, followed by $2.5 \mathrm{ml}$ of saturated $\mathrm{NaCO} 3$ solution. The mixture was diluted $50 \mathrm{ml}$ flask, and incubated for 90 minutes at room temperature. The absorbance was measured at $250 \mathrm{ml}$ in Genway model 6000 specrophometer. The reading was taken with the reagent blank at zero. The tannin content was calculated as follows:

$$
\% \text { Tannin }=\frac{A n / A s \times C \times 100 / V f / V a}{w}
$$

Where, An is the Absorbance of test sample, As is Absorbance of standard solution, $\mathrm{C}$ represents Concentration of standard solution, w is the weight of sample used, $\mathrm{Vf}$ is the Total volume of extract and Va is the Volume of extract analyzed.

Table 3: Formulated Diets of Differently Processed Locust Bean Meal.

\section{Analysis for sapponin content}

This was determined finely ground of dried locust bean seed with a muter and pistil and brewed with hot water at 90 ${ }^{\circ} \mathrm{C}$ for 10 minutes. The obtained locust bean extract was divided into 3 portions containing $20 \mathrm{ml}$ of infusion and treated with $3 \mathrm{ml}$ of $32 \%$ hydrochloric acid solution, in order to yield an acid concentration of $4 \mathrm{~mol} /$ litre prior to hydrolysis for 2 hours. The sapponin fraction was extracted with an equal volume of chloroform using separating funnel. This extraction was performed 3 times. The sapponin was obtained with $10 \mathrm{ml}$ of methanol after evaporation to dryness.

\section{Analysis of hydrocyanic acid content (HCN)}

Table 2: The Anti-nutritive factors in Locust Bean Seed.

\begin{tabular}{|c|c|c|c|c|}
\hline $\begin{array}{c}\text { Anti-Nutritional } \\
\text { Compounds (\%) }\end{array}$ & Raw & Boiled & Fermented & Toasted \\
\hline Saponins & 2.14 & 1.16 & 1.89 & 1.41 \\
\hline Phenols & 19.53 & 7.28 & 14.22 & 10.65 \\
\hline HCN & 1.26 & 0.53 & 0.87 & 0.81 \\
\hline Tanin & 1.46 & 0.48 & 2.46 & 0.66 \\
\hline
\end{tabular}

\begin{tabular}{|c|c|c|c|c|c|c|c|c|c|c|c|c|c|}
\hline \multirow{2}{*}{ Feedstuffs (\%) } & \multirow{2}{*}{$\begin{array}{l}\text { Control } \\
\text { Diet } 1\end{array}$} & \multicolumn{3}{|c|}{$\begin{array}{l}\text { Raw Locust Bean Meal } \\
\text { (RLBM) }\end{array}$} & \multicolumn{4}{|c|}{$\begin{array}{l}\text { Boiled Locust Bean Meal } \\
\text { (BLBM) }\end{array}$} & \multirow{2}{*}{$\begin{array}{c}\begin{array}{c}\text { Fermented } \\
\text { Locust } \\
\text { Bean Meal } \\
\text { (FLBM }\end{array} \\
\text { Diet } 910 \%\end{array}$} & \multicolumn{4}{|c|}{$\begin{array}{l}\text { Toasted Locust Bean Meal } \\
\text { (TLBM }\end{array}$} \\
\hline & & $\begin{array}{l}\text { Diet } 2 \\
\mathbf{5 \%}\end{array}$ & $\begin{array}{c}\text { Diet } 3 \\
10 \%\end{array}$ & $\begin{array}{c}\text { Diet } 4 \\
15 \%\end{array}$ & $\begin{array}{l}\text { Diet } \\
55 \%\end{array}$ & $\begin{array}{l}\text { Diet } 6 \\
10 \%\end{array}$ & $\begin{array}{c}\text { Diet } 7 \\
15 \%\end{array}$ & $\begin{array}{c}\text { Diet } 8 \\
\mathbf{5 \%}\end{array}$ & & $\begin{array}{c}\text { Diet } \\
10 \\
15 \%\end{array}$ & $\begin{array}{c}\text { Diet } \\
\mathbf{1 1} \\
\mathbf{5 \%}\end{array}$ & $\begin{array}{c}\text { Diet } \\
12 \\
10 \%\end{array}$ & $\begin{array}{c}\text { Diet } \\
13 \\
15 \%\end{array}$ \\
\hline Fish meal & 59.94 & 54.28 & 48.9 & 43.76 & 54.11 & 48.57 & 43.33 & 53.78 & 47.93 & 42.43 & 53.79 & 47.96 & 42.47 \\
\hline Maize meal & 32.06 & 32.72 & 33.1 & 33.76 & 32.89 & 33.43 & 33.67 & 33.22 & 34.07 & 34.57 & 33.21 & 34.04 & 34.53 \\
\hline Locust bean & 0 & 5 & 10 & 15 & 5 & 10 & 15 & 5 & 10 & 15 & 5 & 10 & 15 \\
\hline $\begin{array}{l}\text { Vitamin Mineral } \\
\text { premix }\end{array}$ & 5 & 5 & 5 & 5 & 5 & 5 & 5 & 5 & 5 & 5 & 5 & 5 & 5 \\
\hline Oil & 3 & 3 & 3 & 3 & 3 & 3 & 3 & 3 & 3 & 3 & 3 & 3 & 3 \\
\hline Total & 100 & 100 & 100 & 100 & 100 & 100 & 100 & 100 & 100 & 100 & 100 & 100 & 100 \\
\hline \multicolumn{14}{|c|}{ Proximate compositions of formulated diets (\%) } \\
\hline Crude protein & 49.91 & 49.06 & 49.2 & 49.1 & 49.45 & 49.25 & 49.27 & 49.7 & 49.35 & 49.9 & 49.46 & 49.15 & 49.17 \\
\hline Crude fat & 9.92 & 6.86 & 27.9 & 8.13 & 8.27 & 10.69 & 11.22 & 11.61 & 13.8 & 13.3 & 13.3 & 11.74 & 14.86 \\
\hline Crude fiber & 6.81 & 3.86 & 3.92 & 4.14 & 3.33 & 3.57 & 3.72 & 3.88 & 3.89 & 3.96 & 4.7 & 4.84 & 4.96 \\
\hline Ash & 3.64 & 3.89 & 3.94 & 4.16 & 3.44 & 3.88 & 3.98 & 3.88 & 3.96 & 3.99 & 4.08 & 4.26 & 4.87 \\
\hline Dry Matter & 7.36 & 6.52 & 7.66 & 9.23 & 5.89 & 7.8 & 8.17 & 10.15 & 9.26 & 8.38 & 10.35 & 9.44 & 9.2 \\
\hline
\end{tabular}

$20 \mathrm{~g}$ sample of locust bean meal was ground to pass through No. 20 sieve and put in $800 \mathrm{ml}$ kjeldahl flask to which $100 \mathrm{ml}$ water was added. It was macerated at room temperature for 2 hours and $100 \mathrm{ml}$ water was added and steamed to distill, the distillate was collected in $20 \mathrm{ml} 0.02 \mathrm{~N} \mathrm{AgNO}_{3}$ acidified with $1 \mathrm{ml} \mathrm{HNO}_{3}$. Before distillation the tip of condenser was adjusted below the ace of liquid in receiver. When $150 \mathrm{ml}$ has passed over, the distillate was filtered through gooch wash receiver and gooch with little water. Titrate excess $\mathrm{AgNO}_{3}$ in combined filtrate and washing with $0.02 \mathrm{~N}$ KCN, using Fe alum indicators

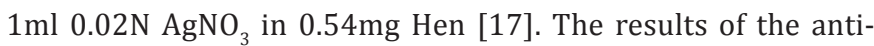
nutritive factors are presented in (Table $2 \& 3$ ). 


\section{Biological evaluations}

Biological parameters evaluated were as according to Maynard et al. [18] \& Halver [19] as described below:

1. Mean Weight Gain (g) was calculated as the difference between mean final carcass weight (g) and mean initial carcass weight $(\mathrm{g})$.

2. Specific Growth Rate (SGR \% Day) lnw2-lnw1/T x 100; $\mathrm{w} 1$ = initial fish weight $(\mathrm{g})$ at time T1 (day), $\mathrm{W} 2$ = final fish weight (g) at time T2 (day) and Ln as natural logarithm.

3. Food Conversion Ratio (FCR) = Feed fed ( $\mathrm{g}$ dry weight)/ Live weight gain (g) Brown [20].

4. Protein Efficiency Ratio (PER) = Live weight gain (g)/ Crude protein fed (g) [21].

5. Apparent Net Protein Utilization $(\mathrm{ANPU})(\%)=(\mathrm{P} 2-$ P1)/Total protein consumed (g) x 100 Bender \& Miller [22], Miller \& Bender [23].

6. Where, P1=Initial carcass protein, P2=final carcass protein.

7. Acid Insoluble Ash (AIA) of the diets and feacal samples were determined according to Cockrell et al. [24].

Table 4: Weekly Water Quality Parameters (Range: Week 0-8).

\begin{tabular}{|c|c|c|c|c|c|c|}
\hline Treatment & $\mathbf{T}^{\circ} \mathbf{C}$ & pH & Conductivity ( $\mu \mathrm{s} / \mathrm{cm})$ & DO (mg/l) & ALK (mg/l) & Hardness (mg/l) \\
\hline Diet1 & $26-30$ & $6.70-7.37$ & $373-427$ & $4.00-6.00$ & $60.10-120$ & 72.71-156 \\
\hline Diet 2 & $26-30$ & $6.71-7.39$ & $368-456$ & $4.60-6.00$ & $66.66-115$ & 82.46-159 \\
\hline Diet 3 & $26-30$ & $6.84-7.26$ & $353-451$ & $4.00-7.50$ & $61.33-121$ & $82.00-165$ \\
\hline Diet 4 & $26-30$ & $6.82-7.64$ & $351-432$ & $4.30-6.00$ & $64-136$ & $77.00-171$ \\
\hline Diet 5 & $26-30$ & $6.90-7.55$ & $358=444$ & $4.30-6.00$ & $63.33-120$ & $82.20-155$ \\
\hline Diet 6 & $26-29$ & $6.66-7.33$ & $375-515$ & $4.00-6.00$ & $63.66-128$ & 87.33-168 \\
\hline Diet 7 & $26-30$ & $6.63-7.50$ & $358-447$ & $4.66-6.00$ & $62.13-138$ & $84.66-173$ \\
\hline Diet 8 & $26-30$ & $6.60-7.43$ & $377-438$ & $4.30-6.00$ & $65.33-123$ & $83.33-167$ \\
\hline Diet 9 & $26-30$ & $6.48-7.43$ & $380-438$ & $4.03-6.30$ & $60.00-126$ & $85.30-165$ \\
\hline Diet 10 & $26-30$ & $6.87-7.59$ & $369-455$ & $4.66-6.00$ & $63.00-118$ & $80.00-155$ \\
\hline Diet 12 & $26-30$ & $6.58-7.64$ & $364-456$ & $4.00-7.33$ & 63.33-138 & $84.00-178$ \\
\hline Diet 13 & $26-30$ & $6.85-7.43$ & $368-442$ & $4.00-6.00$ & $64.23-121$ & $85.30-163$ \\
\hline
\end{tabular}

\section{Results}

Table 5: Growth Response of Clarias gariepinus fingerlings Fed Differently Processed Locust Bean Meal for 56 days.

\begin{tabular}{|c|c|c|c|c|c|c|c|c|c|c|c|c|c|c|}
\hline $\begin{array}{c}\text { Growth } \\
\text { Para- } \\
\text { meters }\end{array}$ & $\begin{array}{c}0 \% \\
\text { LBM) }\end{array}$ & $\begin{array}{c}5 \% \\
\text { RLBM }\end{array}$ & $\begin{array}{c}5 \% \\
\text { BLBM }\end{array}$ & $\begin{array}{c}5 \% \\
\text { FLBM }\end{array}$ & $\begin{array}{c}5 \% \\
\text { TLBM }\end{array}$ & $\begin{array}{c}10 \% \\
\text { RLBM }\end{array}$ & $\begin{array}{c}10 \% \\
\text { BBLBM }\end{array}$ & $\begin{array}{c}10 \% \\
\text { FLBM }\end{array}$ & $\begin{array}{c}10 \% \\
\text { TLBM }\end{array}$ & $\begin{array}{c}15 \% \\
\text { RLBM }\end{array}$ & $\begin{array}{c}15 \% \\
\text { FLBM }\end{array}$ & $\begin{array}{c}15 \% \\
\text { TLBM }\end{array}$ & $\begin{array}{c}15 \% \\
\text { TLBM }\end{array}$ & $\mathrm{SD} \pm$ \\
\hline $\begin{array}{c}\text { Initial } \\
\text { Mean } \\
\text { Weight } \\
\text { Gain (g) }\end{array}$ & $\begin{array}{l}3.14^{\mathrm{b}} \\
\pm 0.05\end{array}$ & $\begin{array}{r}3.83^{\mathrm{a}} \\
\pm 0.78\end{array}$ & $\begin{array}{l}3.76^{\mathrm{a}} \\
\pm 0.67\end{array}$ & $\begin{array}{c}3.36^{b} \\
\pm 0.52\end{array}$ & $\begin{array}{l}3.31^{\mathrm{b}} \\
\pm 0.19\end{array}$ & $\begin{array}{l}3.35^{\mathrm{a}} \\
\pm 0.66\end{array}$ & $\begin{array}{l}3.53^{a} \\
\pm 0.88\end{array}$ & $\begin{array}{l}3.44^{\mathrm{a}} \\
\pm 0.67\end{array}$ & $\begin{array}{l}3.40^{\mathrm{a}} \\
\pm 0.22\end{array}$ & $\begin{array}{l}3.11^{\mathrm{b}} \\
\pm 1.14\end{array}$ & $\begin{array}{l}3.21^{\mathrm{b}} \\
\pm 0.33\end{array}$ & $\begin{array}{l}3.74^{\mathrm{a}} \\
\pm 0.14\end{array}$ & $\begin{array}{l}3.81^{\mathrm{a}} \\
\pm 0.50\end{array}$ & 0.6 \\
\hline $\begin{array}{c}\text { Final } \\
\text { Mean } \\
\text { Weight } \\
\text { Gain (g) }\end{array}$ & $\begin{array}{r}9.57^{\mathrm{a}} \\
\pm 2.78\end{array}$ & $\begin{array}{l}7.87^{b} \\
\pm 0.82\end{array}$ & $\begin{array}{c}8.12^{\mathrm{c}} \\
\pm 1.81\end{array}$ & $\begin{array}{r}6.96^{b} \\
\pm 0.68\end{array}$ & $\begin{array}{c}6.84^{c} \\
\pm 0.32\end{array}$ & $\begin{array}{l}8.05^{\mathrm{ab}} \\
\pm 0.49\end{array}$ & $\begin{array}{l}8.53^{\mathrm{b}} \\
\pm 0.54\end{array}$ & $\begin{array}{r}9.08^{\mathrm{a}} \\
\pm 1.23\end{array}$ & $\begin{array}{l}8.40^{\mathrm{b}} \\
\pm 0.22\end{array}$ & $\begin{array}{c}8.12^{\mathrm{c}} \\
\pm 0.46\end{array}$ & $\begin{array}{l}8.84^{\mathrm{b}} \\
\pm 0.35\end{array}$ & $\begin{array}{c}8.17^{c} \\
\pm 0.14\end{array}$ & $\begin{array}{c}8.67^{a} \\
\pm 0.28\end{array}$ & 0.9 \\
\hline
\end{tabular}


Oceanography \& Fisheries Open access Journal

\begin{tabular}{|c|c|c|c|c|c|c|c|c|c|c|c|c|c|c|}
\hline $\begin{array}{c}\text { Mean } \\
\text { Weight } \\
\text { Gain (g) }\end{array}$ & $\begin{array}{r}6.44 \mathrm{a} \\
\pm 2.78\end{array}$ & $\begin{array}{l}4.05_{\mathrm{f}} \\
\pm 0.28\end{array}$ & $\begin{array}{l}4.34 \mathrm{e} \\
\pm 2.03\end{array}$ & $\begin{array}{l}5.84^{\mathrm{b}} \\
\pm 1.08\end{array}$ & $\begin{array}{l}5.47^{\mathrm{bc}} \\
\pm 1.34\end{array}$ & $\begin{array}{l}4.70^{\text {de }} \\
\pm 1.12\end{array}$ & $\begin{array}{l}5.01^{\mathrm{d}} \\
\pm 1.12\end{array}$ & $\begin{array}{l}5.64^{c} \\
\pm 0.78\end{array}$ & $\begin{array}{l}5.00^{\mathrm{d}} \\
\pm 0.34\end{array}$ & $\begin{array}{l}5.01^{\mathrm{c}} \\
\pm 1.19\end{array}$ & $\begin{array}{l}5.13^{\mathrm{d}} \\
\pm 0.38\end{array}$ & $\begin{array}{l}4.42^{\mathrm{d}} \\
\pm 0.31\end{array}$ & $\begin{array}{l}4.87^{\mathrm{d}} \\
\pm 0.66\end{array}$ & 1.1 \\
\hline $\begin{array}{c}\text { Specific } \\
\text { Growth } \\
\text { Rate } \\
\text { (\%/ } \\
\text { Day) }\end{array}$ & $\begin{array}{l}2.14 \mathrm{a} \\
\pm 0.80\end{array}$ & $\begin{array}{l}1.33_{c} \\
\pm 0.58\end{array}$ & $\begin{array}{l}1.36 \mathrm{c} \\
\pm 0.55\end{array}$ & $\begin{array}{l}2.00 \\
\pm 0.00\end{array}$ & $\begin{array}{l}1.67^{\mathrm{b}} \\
\pm 0.58\end{array}$ & $\begin{array}{l}1.33^{c} \\
\pm 0.58\end{array}$ & $\begin{array}{l}1.33^{c} \\
\pm 0.58\end{array}$ & $\begin{array}{l}2.00^{\mathrm{a}} \\
\pm 0.00\end{array}$ & $\begin{array}{l}1.67^{\mathrm{b}} \\
\pm 0.58\end{array}$ & $\begin{array}{l}1.62^{\mathrm{b}} \\
\pm 0.54\end{array}$ & $\begin{array}{l}2.00^{\mathrm{a}} \\
\pm 0.00\end{array}$ & $\begin{array}{l}1.33^{\mathrm{c}} \\
\pm 0.58\end{array}$ & $\begin{array}{l}1.33^{\mathrm{c}} \\
\pm 0.58\end{array}$ & 0.3 \\
\hline $\begin{array}{c}\text { Feed } \\
\text { Conv- } \\
\text { ersion } \\
\text { Ratio }\end{array}$ & $\begin{array}{l}1.17_{\mathrm{d}} \\
\pm 0.39\end{array}$ & $\begin{array}{l}1.51_{\mathrm{b}} \\
\pm 0.17\end{array}$ & $\begin{array}{l}1.68 \mathrm{a} \\
\pm 0.53\end{array}$ & $\begin{array}{l}1.02_{\mathrm{d}} \\
\pm 0.24\end{array}$ & $\begin{array}{l}1.13^{\mathrm{d}} \\
\pm 0.23\end{array}$ & $\begin{array}{l}1.60^{\mathrm{a}} \\
\pm 0.26\end{array}$ & $\begin{array}{l}1.24^{\mathrm{bc}} \\
\pm 0.10\end{array}$ & $\begin{array}{l}1.28^{c} \\
\pm 0.36\end{array}$ & $\begin{array}{l}1.21^{\mathrm{bc}} \\
\pm 0.36\end{array}$ & $\begin{array}{l}1.10^{\mathrm{d}} \\
\pm 0.15\end{array}$ & $\begin{array}{l}1.43^{\mathrm{c}} \\
\pm 0.10\end{array}$ & $\begin{array}{l}1.23^{\mathrm{bc}} \\
\pm 0.53\end{array}$ & $\begin{array}{l}1.34^{\mathrm{b}} \\
\pm 0.25\end{array}$ & 0.5 \\
\hline $\begin{array}{l}\text { Protein } \\
\text { Effici- } \\
\text { ency } \\
\text { Ratio }\end{array}$ & $\begin{array}{l}1.86_{b} \\
\pm 0.68\end{array}$ & $\begin{array}{l}1.51_{c} \\
\pm 0.14\end{array}$ & $\begin{array}{l}1.41 \mathrm{~d} \\
\pm 0.59\end{array}$ & $\begin{array}{l}2.16+ \\
\pm 0.64\end{array}$ & $\begin{array}{l}1.84^{\mathrm{b}} \\
\pm 0.52\end{array}$ & $\begin{array}{l}1.45^{\mathrm{d}} \\
\pm 0.25\end{array}$ & $\begin{array}{l}1.68^{c} \\
\pm 0.14\end{array}$ & $\begin{array}{l}1.91^{\mathrm{b}} \\
\pm 0.62\end{array}$ & $\begin{array}{l}1.91^{\mathrm{b}} \\
\pm 0.62\end{array}$ & $\begin{array}{l}1.99^{\mathrm{b}} \\
\pm 0.26\end{array}$ & $\begin{array}{l}1.50^{\mathrm{d}} \\
\pm 0.17\end{array}$ & $\begin{array}{c}1.98^{\mathrm{b}} \\
\pm 0.90\end{array}$ & $\begin{array}{l}1.58^{\mathrm{c}} \\
\pm 0.32\end{array}$ & 0.5 \\
\hline $\begin{array}{l}\text { Appa- } \\
\text { rent } \\
\text { Net } \\
\text { Protein } \\
\text { Utiliz- } \\
\text { ation } \\
(\%)\end{array}$ & $\begin{array}{l}98.40_{c} \\
\pm 8.40^{\circ}\end{array}$ & $\begin{array}{l}134.73_{\mathrm{b}} \\
\pm 16.93\end{array}$ & $\begin{array}{l}98.78^{c} \\
\pm 4.26\end{array}$ & $\begin{array}{c}178.31^{\mathrm{a}} \\
\pm 6.89\end{array}$ & $\begin{array}{l}168.0^{\mathrm{a}} \\
\pm 15.08\end{array}$ & $\begin{array}{c}122 . \\
91^{\text {ab }} \\
\pm 8.94\end{array}$ & $\begin{array}{c}171.29^{\mathrm{a}} \\
\pm 8.81\end{array}$ & $\begin{array}{c}160 \\
.17^{\mathrm{a}} \\
\pm 28.80\end{array}$ & $\begin{array}{l}160.17^{\mathrm{a}} \\
\pm 47.65\end{array}$ & $\begin{array}{l}29.83^{\mathrm{b}} \\
\pm 6.93\end{array}$ & $\begin{array}{r}124 \\
.63^{\mathrm{a}} \\
\pm 3.35\end{array}$ & $\begin{array}{r}85.20^{\mathrm{d}} \\
\pm 73.58\end{array}$ & $\begin{array}{l}86.05^{\mathrm{d}} \\
\pm 5.75\end{array}$ & 26 \\
\hline $\begin{array}{l}\text { Morta- } \\
\text { lity (\%) }\end{array}$ & 28.9 & 24.4 & 22.2 & 35.6 & 33.3 & 17.8 & 33.3 & 31.1 & 42.2 & 37.8 & 17.8 & 37.8 & 31.1 & \\
\hline
\end{tabular}

Data on the same row carrying similar superscripts are not significantly different from each other $(P>0.05)$.

The results showed in Table 5 on the growth parameters exhibited significant differences $(\mathrm{p}<0.05)$ among the treatments. The control diet with $0 \%$ locust bean meal $(0 \% \mathrm{LBM})$, gave a significantly highest $(\mathrm{P}<0.05)$ mean weight gain $(\mathrm{MWG})$ of $6.44 \mathrm{~g}$ which was closely followed by $5 \%$ fermented locust bean meal (FLBM) and 5\% toasted locust bean meal (TLBM) diets with average mean weight gains of $5.84 \mathrm{~g}$ and $5.47 \mathrm{~g}$ respectively which are also significantly different $(\mathrm{P}<0.05)$ from each other. While $5 \%$ boiled and raw locust bean meal diets recorded significantly low $(\mathrm{P}<0.05) \mathrm{MWG}$ of $4.34 \mathrm{~g}$ and $4.05 \mathrm{~g}$ which are significantly different $(\mathrm{P}<0.05)$ from each other. As inclusion level was adjusted to $10 \%$, the fermented locust bean diet still performed best $(5.64 \mathrm{~g}$ ) followed by boiled and toasted with MWG values of $5.01 \mathrm{~g}$ and $5.00 \mathrm{~g}$ which were not significantly different $(\mathrm{P}>0.05)$ from each other. At the highest inclusion level of $15 \%$, the boiled locust bean meal diet significantly did better (5.13g) than other diets, however its performance was followed by raw locust bean meal diet (5.01g) which was followed by the toasted locust bean meal diet (4.87g) and the least performed diet was the fermented locust bean meal (4.42g).

The specific growth rate (SGR) for the control diet followed the same trend as observed with mean weight gain with the control diet still exhibited the highest value of $2.14 \%$ which was significantly different $(\mathrm{P}<0.05)$ from other diets. Moreover, $5 \%$ fermented locust bean diet had the best SGR value (2.00) which was followed by the toasted diet (1.67) while the cooked (1.36) and raw (1.33) locust bean meal diets had the lowest values and were not significantly different $(\mathrm{P}>0.05)$ from each other. The $10 \%$ inclusive level followed similar trend as $5 \%$ inclusion level. Moreover, as the inclusion level hit the highest level of $15 \%$, the cooked locust bean based diet gave SGR value of 2.00 which was significantly higher than the raw locust bean diet (1.62) which was also significantly higher than those of fermented (1.33) and toasted (1.33) locust bean based diets which were not significantly different $(\mathrm{P}>0.05)$ from each other.

The best feed conversion ratio (FCR) value obtained at 5\% inclusion level was from the fermented locust bean diet (1.02) which was followed by toasted (1.13) and the control diet (1.17) while the raw locust bean meal diets gave the poorest value of 1.51. Moreover, at $10 \%$ inclusion level, the toasted locust bean diet exhibited its best value (1.21), followed by the cooked locust bean diet (1.24), fermented diet (1.28) while the raw gave the poorest value of 1.60 . When the inclusion level reached $15 \%$ the raw locust bean meal diet gave the best value of 1.10 , followed by fermented locust bean diet (1.23), toasted (1.34) while the cooked locust bean diet recorded the poorest value (1.43).

The protein efficiency ratio (PER) value indicated significant difference $(\mathrm{P}<0.05)$ for the experimental diets. At $5 \%$ inclusion level, the fermented locust bean diet gave a significantly high $(\mathrm{P}<0.05)$ value $(178.21 \%)$ followed by the toasted locust bean diet $(168.0 \%)$, and then the raw diet (134.73\%) while the cooked and the control diet recorded significantly low values (98.78\% and $98.40 \%)$ respectively. However, as the inclusion level increased to $10 \%$, the cooked and toasted locust bean diets performed best with ANPU values of $160.17 \%$ each which was followed by the raw locust bean diet (122.91\%) which was significantly higher $(\mathrm{P}<0.05)$ than the control diet $(98.40 \%)$. Furthermore, at the highest inclusion level of $15 \%$, the cooked performed best $(124.6 \%)$ a value that was significantly higher $(\mathrm{P}<0.05)$ than those of toasted $(86.05 \%)$, and fermented 
$(85.20 \%)$ which were not significantly different $(\mathrm{P}>0.05)$ from each other but significantly different from $(\mathrm{P}<0.05)$ from the control diet $(98.40 \%)$ while the raw locust bean diet gave the poorest value (29.88\%).

The mortality rates of the fishes were significantly high $(\mathrm{P}<0.05)$ for diet containing $10 \%$ locust bean meal $(42.2 \%)$ while the lowest mortality rate of $17.8 \%$ were observed for both $10 \%$ raw locust bean meal and $15 \%$ cooked locust bean meal while all others were at their optimum level. The proximate body compositions of Clarias gariepinus fed locust bean meal that were subjected to different processing methods indicated significant differences among the treatments $(\mathrm{P}<0.05)$ as shown in Table 6 . The values obtained at $5 \%$ inclusion level of various diets showed positive muscle deposit on the fish compared with the initial value (62.62\%). The toasted locust bean meal diet gave a significantly high $(\mathrm{P}<0.05)$ crude protein value $(67.37 \%)$ and was followed by the raw locust bean meal diet (66.50\%) which was not significantly different $(\mathrm{P}>0.05)$ from that of the boiled locust bean diet $(65.62 \%)$ as well as the control diet $(65.92 \%)$ but were significantly different $(\mathrm{P}<0.05)$ from that of fermented locust bean meal diet (63.87\%) which was equally significantly different $(\mathrm{P}<0.05)$ from the initial body protein value $(62.62 \%)$.

Table 6: Proximate Composition of Clarias gariepinus fingerlings Fed Differently Processed Locust Bean Meal for 56 days.

\begin{tabular}{|c|c|c|c|c|c|c|c|c|c|c|c|c|c|c|c|}
\hline $\begin{array}{l}\text { Prox- } \\
\text { imate } \\
\text { compo- } \\
\text { sitions } \\
(\%)\end{array}$ & Initial & $\begin{array}{c}0 \% \\
\text { LBM }\end{array}$ & $\begin{array}{c}\mathbf{5 \%} \\
\text { RLBM }\end{array}$ & $\begin{array}{c}5 \% \\
\text { BLBM }\end{array}$ & $\begin{array}{c}5 \% \\
\text { FLBM }\end{array}$ & $\begin{array}{c}5 \% \\
\text { TLBM }\end{array}$ & $\begin{array}{c}10 \% \\
\text { RLBM }\end{array}$ & $\begin{array}{c}10 \% \\
\text { BLBM }\end{array}$ & $\begin{array}{c}10 \% \\
\text { FLBM }\end{array}$ & $\begin{array}{c}10 \% \\
\text { TLBM }\end{array}$ & $\begin{array}{c}15 \% \\
\text { RLBM }\end{array}$ & $\begin{array}{c}15 \% \\
\text { BLBM }\end{array}$ & $\begin{array}{c}15 \% \\
\text { FLBM }\end{array}$ & $\begin{array}{c}15 \% \\
\text { TLBM }\end{array}$ & $\begin{array}{l}\text { SD } \\
\pm\end{array}$ \\
\hline $\begin{array}{l}\text { Crude } \\
\text { Protein }\end{array}$ & $\begin{array}{l}62.62^{\mathrm{c}} \\
\pm 0.01\end{array}$ & $\begin{array}{l}65.97^{b} \\
\pm 0.01\end{array}$ & $\begin{array}{c}66.50^{\mathrm{b}} \\
\pm 0.01\end{array}$ & $\begin{array}{l}65.62^{\mathrm{b}} \\
\pm 0.01\end{array}$ & $\begin{array}{l}63.87^{c} \\
\pm 0.01\end{array}$ & $\begin{array}{l}67.37^{\mathrm{a}} \\
\pm 0.01\end{array}$ & $\begin{array}{l}68.66^{a} \\
\pm 0.01\end{array}$ & $\begin{array}{c}64.70 \mathrm{~b}^{\mathrm{c}} \\
\pm 0.01\end{array}$ & $\begin{array}{l}61.83^{\mathrm{d}} \\
\pm 0.01\end{array}$ & $\begin{array}{l}66.40^{\mathrm{b}} \\
\pm 0.01\end{array}$ & $\begin{array}{l}63.35^{\mathrm{c}} \\
\pm 1.01\end{array}$ & $\begin{array}{l}67.00^{\mathrm{a}} \\
\pm 1.01\end{array}$ & $\begin{array}{l}58.00^{\mathrm{d}} \\
\pm 1.01\end{array}$ & $\begin{array}{c}65.28^{\mathrm{b}} \\
\pm 1.01\end{array}$ & 0.01 \\
\hline $\begin{array}{c}\text { Crude } \\
\text { FAT }\end{array}$ & $\begin{array}{l}5.10^{c} \\
\pm 0.01\end{array}$ & $\begin{array}{l}10.99^{\mathrm{a}} \\
\pm 0.06\end{array}$ & $\begin{array}{l}10.95^{\mathrm{a}} \\
\pm 0.01\end{array}$ & $\begin{array}{c}9.17^{\mathrm{b}} \\
\pm 0.01\end{array}$ & $\begin{array}{l}8.34^{\mathrm{c}} \\
\pm 0.01\end{array}$ & $\begin{array}{l}8.61^{\mathrm{c}} \\
\pm 0.01\end{array}$ & $\begin{array}{l}9.00^{\mathrm{b}} \\
\pm 0.01\end{array}$ & $\begin{array}{l}8.96^{\mathrm{b}} \\
\pm 0.01\end{array}$ & $\begin{array}{l}8.07^{\mathrm{c}} \\
\pm 0.05\end{array}$ & $\begin{array}{l}9.45^{\mathrm{b}} \\
\pm 0.46\end{array}$ & $\begin{array}{l}9.20^{\mathrm{b}} \\
\pm 1.01\end{array}$ & $\begin{array}{l}10.20^{\mathrm{b}} \\
\pm 1.01\end{array}$ & $\begin{array}{l}10.10^{\mathrm{b}} \\
\pm 1.01\end{array}$ & $\begin{array}{c}10.04^{\mathrm{b}} \\
\pm 1.01\end{array}$ & 0.01 \\
\hline ASH & $\begin{array}{l}4.45^{\mathrm{b}} \\
\pm 0.01\end{array}$ & $\begin{array}{l}8.98^{\mathrm{ab}} \\
\pm 0.01\end{array}$ & $\begin{array}{c}9.45^{\mathrm{a}} \\
\pm 0.01\end{array}$ & $\begin{array}{c}9.70^{\mathrm{a}} \\
\pm 0.01\end{array}$ & $\begin{array}{l}9.17 a \\
\pm 0.01\end{array}$ & $\begin{array}{l}9.47^{\mathrm{a}} \\
\pm 0.01\end{array}$ & $\begin{array}{l}9.79^{\mathrm{a}} \\
\pm 0.01\end{array}$ & $\begin{array}{l}10.19^{\mathrm{a}} \\
\pm 0.01\end{array}$ & $\begin{array}{c}9.30^{\mathrm{a}} \\
\pm 0.01\end{array}$ & $\begin{array}{l}9.65^{\mathrm{a}} \\
\pm 0.01\end{array}$ & $\begin{array}{l}7.98^{\mathrm{c}} \\
\pm 1.01\end{array}$ & $\begin{array}{l}10.15^{\mathrm{a}} \\
\pm 1.01\end{array}$ & $\begin{array}{l}9.80^{\mathrm{b}} \\
\pm 1.01\end{array}$ & $\begin{array}{c}9.35^{\mathrm{b}} \\
\pm 1.01\end{array}$ & 0.01 \\
\hline $\begin{array}{c}\text { Crude } \\
\text { Fibre }\end{array}$ & $\begin{array}{l}19.26 \\
\pm 0.01\end{array}$ & $\begin{array}{c}6.84 \\
\pm 0.01\end{array}$ & $\begin{array}{c}2.90^{\mathrm{b}} \\
\pm 0.01\end{array}$ & $\begin{array}{c}6.60 \\
\pm 0.01\end{array}$ & $\begin{array}{c}8.78 \\
\pm 0.01\end{array}$ & $\begin{array}{c}4.34 \\
\pm 0.01\end{array}$ & $\begin{array}{c}4.12 \\
\pm 0.01\end{array}$ & $\begin{array}{c}8.82 \\
\pm 0.01\end{array}$ & $\begin{array}{l}13.62 \\
\pm 0.01\end{array}$ & $\begin{array}{c}6.89 \\
\pm 0.01\end{array}$ & $\begin{array}{c}9.77 \\
\pm 0.01\end{array}$ & $\begin{array}{c}4.16 \\
\pm 0.01\end{array}$ & $\begin{array}{l}11.34 \\
\pm 0.01\end{array}$ & $\begin{array}{c}6.93 \\
\pm 0.01\end{array}$ & 0.16 \\
\hline $\begin{array}{l}\text { Mois- } \\
\text { ture } \\
\text { Con- } \\
\text { tent }\end{array}$ & $\begin{array}{l}8.57^{\mathrm{b}} \\
+0.01\end{array}$ & $\begin{array}{c}7.20^{c} \\
+0.01\end{array}$ & $\begin{array}{l}10.20^{\mathrm{a}} \\
\pm 0.02\end{array}$ & $\begin{array}{l}8.91^{\mathrm{b}} \\
\pm 0.01\end{array}$ & $\begin{array}{l}9.81^{\mathrm{ab}} \\
\pm 0.01\end{array}$ & $\begin{array}{l}10.19^{\mathrm{a}} \\
\pm 0.01\end{array}$ & $\begin{array}{l}8.43^{\mathrm{a}} \\
\pm 0.01\end{array}$ & $\begin{array}{l}7.33^{\mathrm{b}} \\
\pm 0.01\end{array}$ & $\begin{array}{l}7.20^{\mathrm{b}} \\
\pm 0.01\end{array}$ & $\begin{array}{l}7.15= \\
\pm 0.01\end{array}$ & $\begin{array}{l}9.70^{\mathrm{a}} \\
\pm 1.01\end{array}$ & $\begin{array}{l}8.40^{\mathrm{b}} \\
\pm 1.01\end{array}$ & $\begin{array}{c}9.86^{\mathrm{a}} \\
\pm 1.01\end{array}$ & $\begin{array}{l}8.40^{\mathrm{b}} \\
\pm 1.01\end{array}$ & 0.15 \\
\hline
\end{tabular}

Data on the same row carrying similar superscripts are not significantly different from each other $(P>0.05)$.

The $10 \%$ inclusion level exhibited best crude protein value for the raw locust bean meal diet (68.60\%) followed by the toasted diet (66.40\%), boiled locust bean diet (64.70\%), initial value of $62.62 \%$ while the fermented locust bean diet had a significantly low $\mathrm{P}(0.05)$ value of $61.83 \%$. As the inclusion level heightened to $15 \%$, the boiled locust bean meal diet performed the best $(67.00 \%)$ followed by toasted diet $(65.28 \%)$, the roasted locust bean diet $(63.35 \%)$, the initial $(62.62 \%)$ while the fermented similarly gave the lowest value of $58.00 \%$. The body fat also indicated significant differences $(\mathrm{P}<0.05)$ among the treatments. When the inclusion level was at $5 \%$, the control and the raw locust bean meal diets gave the highest body fat of $10.99 \%$ and $10.95 \%$ respectively which higher than those of boiled (9.17\%), the toasted diet (8.64\%) which was not significantly different $(\mathrm{P}>0.05)$ from that of fermented locust bean diet $(8.34 \%)$ but significantly different $(\mathrm{P}<0.05)$ from the initial carcass value $(5.10 \%)$.

At $10 \%$ inclusion level, the control diet exhibited a significantly high $\mathrm{P}<0.05)$ body fat value $(10.99 \%)$ than those of toasted locust bean meal diet (8.61\%) which was not significantly different $(\mathrm{P}>0.05)$ from the boiled locust bean meal diet $(8.96 \%)$ but significantly different $(\mathrm{P}<0.05)$ from the fermented locust bean diet $(8.07 \%)$ whose value was significantly different $(\mathrm{P}<0.05)$ from that of the initial body fat. At $15 \%$ inclusion level, the control diet still had the highest body fat $(10.99 \%)$ which was significantly different $(\mathrm{P}<0.05)$ from the toasted diet (10.04\%) and the boiled diet (10.20\%) which were not significantly different $(\mathrm{P}>0.05)$ from each other but significantly different $(\mathrm{P}<0.05)$ from that of raw locust bean diet (9.20\%) while the lowest body fat was recorded for the initial carcass $(5.10 \%)$. The body minerals was significantly high for boiled locust bean diet (9.70\%) at 5\% inclusion level which was not significantly different from those of toasted diet $(9.47 \%)$, fermented diet $(9.45 \%)$ which were significantly different from the control diet (8.98\%) and the initial body ash value (5.10).

As inclusion level increased to $10 \%$, the ash value was still significantly high $(\mathrm{P}<0.05)$ for the boiled diet $(10.19 \%)$, followed by toasted diet $(9.65 \%)$ which was not significantly different $(\mathrm{P}>0.05)$ from the fermented diet $(9.30 \%)$, but were significantly different $(\mathrm{P}<0.05)$ from the control diet $(8.98 \%)$ which is also significantly different $(\mathrm{P}<0.05)$ from the initial carcass value $(4.45 \%)$. The fibre content of the carcass also indicated significant difference $(\mathrm{P}<0.05)$ among the treatments. And of the $5 \%$ inclusion diets, the initial carcass value $(19.26 \%)$ was significantly higher $(\mathrm{P}<0.05)$ than fermented diet $(8.78 \%)$ and the control diet $(6.84 \%)$ which was not significantly different 
$(\mathrm{P}>0.05)$ from the boiled locust bean diet $(6.60 \%)$ but was significantly different $(\mathrm{P}<0.05)$ from that of the roasted locust bean meal diet (4.34\%) while the raw diet had a significantly low $(\mathrm{P}<0.05)$ value $(2.90 \%)$. For the $10 \%$ inclusion level, the initial still had a significantly high fibre value $(19.26 \%)$ than the fermented diet (13.62\%), boiled diet (8.82\%) and toasted $\operatorname{diet}(6.69 \%)$ which was not significantly different $(\mathrm{P}>0.05)$ from the control diet (6.84\%) and the raw locust bean diet (4.12\%).
Furthermore, at $15 \%$ inclusion level, the initial carcass value $(19.26 \%)$ was significantly high $(\mathrm{P}<0.05)$ than other nutrients including the fermented diet (11.34\%0, raw diet (9.77\%) which are significantly different from $(\mathrm{P}<0.05)$ the toasted locust bean $\operatorname{diet}(6.93 \%)$, the control diet (6.84\%) and the boiled locust bean diet $(4.16 \%)$. Table 7 showed the results of digestibility of the experimental diets fed to Clarias gariepinus which indicated significant differences $(\mathrm{P}<0.05)$ among the treatments.

Table 7: Apparent Digestibility Coefficient of differently Processed Methods of Locust Bean Meal Fed to Clarias gariepinus fingerlings for 56 days.

\begin{tabular}{|c|c|c|c|c|c|c|c|c|c|c|c|c|c|c|}
\hline $\begin{array}{l}\text { ADC } \\
(\%)\end{array}$ & $\begin{array}{c}0 \% \\
\text { LBM }\end{array}$ & $\begin{array}{c}\mathbf{5 \%} \\
\text { RLBM }\end{array}$ & $\begin{array}{c}5 \% \\
\text { BLBM }\end{array}$ & $\begin{array}{c}\mathbf{5 \%} \\
\text { FLBM }\end{array}$ & $\begin{array}{c}\mathbf{5 \%} \\
\text { TLBM }\end{array}$ & $\begin{array}{c}10 \% \\
\text { RLBM }\end{array}$ & $\begin{array}{c}10 \% \\
\text { BLBM }\end{array}$ & $\begin{array}{c}10 \% \\
\text { TLBM }\end{array}$ & $\begin{array}{c}10 \% \\
\text { FLBM }\end{array}$ & $\begin{array}{c}15 \% \\
\text { RLBM }\end{array}$ & $\begin{array}{c}15 \% \\
\text { BLBM }\end{array}$ & $\begin{array}{c}\mathbf{1 5} \% \\
\text { TLBM }\end{array}$ & $\begin{array}{c}\mathbf{1 5 \%} \% \\
\text { FLBM }\end{array}$ & $\begin{array}{l}\text { SD } \\
\pm\end{array}$ \\
\hline $\mathrm{CP}$ & $\begin{array}{c}77.51^{\mathrm{a}}+ \\
0.06\end{array}$ & $\begin{array}{l}63.10^{\mathrm{c}} \\
+0.02\end{array}$ & $\begin{array}{c}52.80^{\mathrm{d}} \\
+0.01\end{array}$ & $\begin{array}{l}63.30^{\mathrm{c}} \\
+0.01\end{array}$ & $\begin{array}{c}73.80^{\mathrm{a}} \\
+0.01\end{array}$ & $\begin{array}{l}60.70^{c} \\
+0.01\end{array}$ & $\begin{array}{l}61.70^{c} \\
+0.01\end{array}$ & $\begin{array}{l}66.40^{\mathrm{b}} \\
+0.01\end{array}$ & $\begin{array}{l}62.80^{c} \\
+0.01\end{array}$ & $\begin{array}{l}59.60^{c} \\
+1.01\end{array}$ & $\begin{array}{c}58.90^{\mathrm{c}} \\
+1.01\end{array}$ & $\begin{array}{l}60.90^{\mathrm{c}} \\
+1.01\end{array}$ & $\begin{array}{l}60.80^{c} \\
+1.01\end{array}$ & 0.74 \\
\hline FAT & $\begin{array}{l}66.00^{\mathrm{a}} \\
+0.06\end{array}$ & $\begin{array}{c}50.50^{\mathrm{bc}} \\
+0.01\end{array}$ & $\begin{array}{l}41.50^{\mathrm{d}} \\
+0.01\end{array}$ & $\begin{array}{l}59.30^{c} \\
+0.01\end{array}$ & $\begin{array}{l}64.90^{\mathrm{b}} \\
+0.01\end{array}$ & $\begin{array}{l}44.30^{\mathrm{d}} \\
+0.01\end{array}$ & $\begin{array}{c}45.20^{\mathrm{d}} \\
+0.01\end{array}$ & $\begin{array}{l}67.36^{a} \\
+0.46\end{array}$ & $\begin{array}{l}62.73^{b} \\
+0.05\end{array}$ & $\begin{array}{c}38.50^{\text {cd }} \\
+1.01\end{array}$ & $\begin{array}{c}58.90^{\mathrm{b}} \\
+1.01\end{array}$ & $\begin{array}{c}56.30^{\mathrm{b}} \\
+1.01\end{array}$ & $\begin{array}{l}48.60^{c} \\
+1.01\end{array}$ & 0.08 \\
\hline FIBRE & $\begin{array}{c}31.90^{\mathrm{bc}} \\
+0.01\end{array}$ & $\begin{array}{c}31.89^{\mathrm{bc}} \\
+0.01\end{array}$ & $\begin{array}{l}87.90^{\mathrm{a}} \\
+0.01\end{array}$ & $\begin{array}{c}32.50^{\mathrm{bc}} \\
+0.01\end{array}$ & $\begin{array}{c}28.40^{\mathrm{d}} \\
+0.01\end{array}$ & $\begin{array}{l}21.02^{\mathrm{d}} \\
+0.01\end{array}$ & $\begin{array}{l}59.60^{\mathrm{a}} \\
+0.01\end{array}$ & $\begin{array}{c}26.80^{\mathrm{d}} \\
+0.01\end{array}$ & $\begin{array}{c}33.90^{\mathrm{bc}} \\
+0.01\end{array}$ & $\begin{array}{c}31.10^{\mathrm{bc}} \\
+1.01\end{array}$ & $\begin{array}{l}45.90^{\mathrm{b}} \\
+1.01\end{array}$ & $\begin{array}{c}37.90^{\mathrm{b}} \\
+1.01\end{array}$ & $\begin{array}{c}37.40^{\mathrm{b}} \\
+1.01\end{array}$ & 0.01 \\
\hline ASH & $\begin{array}{c}20.40 \mathrm{~g} \\
+0.01\end{array}$ & $\begin{array}{l}74.30^{c} \\
+0.01\end{array}$ & $\begin{array}{l}60.50^{\mathrm{d}} \\
+0.01\end{array}$ & $\begin{array}{c}84.89^{b} \\
+0.01\end{array}$ & $\begin{array}{c}96.03^{\mathrm{a}} \\
+0.01\end{array}$ & $\begin{array}{c}24.80 \mathrm{~g} \\
+0.01\end{array}$ & $\begin{array}{c}40.40^{\mathrm{e}} \\
+0.01\end{array}$ & $\begin{array}{c}49.40^{\mathrm{e}} \\
+0.01\end{array}$ & $\begin{array}{l}54.90^{\mathrm{d}} \\
+0.01\end{array}$ & $\begin{array}{c}49.40^{\mathrm{e}} \\
+1.01\end{array}$ & $\begin{array}{l}37.70^{\mathrm{f}} \\
+1.01\end{array}$ & $\begin{array}{l}35.50^{\mathrm{f}} \\
+1.01\end{array}$ & $\begin{array}{c}40.90^{\mathrm{e}} \\
+1.01\end{array}$ & 0.01 \\
\hline $\begin{array}{c}\mathrm{DM} \\
(\mathrm{MC})\end{array}$ & $\begin{array}{l}60.30^{\mathrm{a}} \\
+0.01\end{array}$ & $\begin{array}{l}48.10^{\mathrm{b}} \\
+0.02\end{array}$ & $\begin{array}{l}18.99^{\mathrm{d}} \\
+0.01\end{array}$ & $\begin{array}{l}49.40^{\mathrm{b}} \\
+0.01\end{array}$ & $\begin{array}{l}51.90^{\mathrm{a}} \\
+0.01\end{array}$ & $\begin{array}{l}56.8^{0 \mathrm{a}} \\
+0.01\end{array}$ & $\begin{array}{l}35.60^{c} \\
+0.01\end{array}$ & $\begin{array}{c}58.90^{\mathrm{a}} \\
+0.01\end{array}$ & $\begin{array}{l}55.96^{\mathrm{a}} \\
+0.01\end{array}$ & $\begin{array}{c}38.40^{c} \\
+1.01\end{array}$ & $\begin{array}{l}52.20^{\mathrm{a}} \\
+1.01\end{array}$ & $\begin{array}{c}38.80^{c} \\
+1.01\end{array}$ & $\begin{array}{l}41.60^{\mathrm{b}} \\
+1.01\end{array}$ & 0.02 \\
\hline
\end{tabular}

Data on the same row carrying similar superscripts are not significantly different from each other $(P>0.05)$.

The crude protein digestibility at $5 \%$ inclusion level was highest for the control diet $(77.51 \%)$ followed by the toasted locust bean diet $(73.80 \%)$, the raw diet $(63.10 \%)$ which was not significantly different $(\mathrm{P}>0.05)$ from the fermented diet $(63.30 \%)$ but was significantly different $(\mathrm{P}>0.05)$ from the boiled $\operatorname{diet}(52.80 \%)$. When the inclusion level was increased to $10 \%$, the control diet was significantly higher $(\mathrm{P}<0.05)(77.51 \%)$ than the toasted diet $(66.40 \%)$, the fermented diet (62.80\%) which was not significantly different from the boiled diet (61.70\%) and the raw diet $(60.70 \%)$. Similarly, at $15 \%$ the control diet still performed best in terms of digestibility with $77.51 \%$ than the toasted diet $(60.90 \%)$ which was not significantly different $(\mathrm{P}>0.05)$ from the fermented diet $(60.80 \%)$, the boiled diet $(50.90 \%)$ which was not significantly different $(\mathrm{P}>0.05)$ from the raw locust bean diet (59.60\%).

The fat digestibility at $5 \%$ inclusion level gave the best value for the control diet $(66.0 \%)$ which was not significantly different $(\mathrm{P}>0.05)$ from the toasted diet $(64.90 \%)$, but significantly different $(\mathrm{P}<0.05)$ from fermented diet $(59.30 \%)$, the raw diet $(50.50 \%)$ while the boiled gave a significantly low value (41.50\%). With increment to $10 \%$, the toasted diet (67.36\%), the control diet $(66.0 \%)$ which are not significantly different from each other $(\mathrm{P}>0.05)$ but significantly different $(\mathrm{P}<0.05)$ from the fermented diet (62.77\%). However, the boiled and the raw diets had a significantly low digestibility values ( $45.20 \%$ and $44.30 \%)$ respectively which are not significantly different $(\mathrm{P}>0.05)$ from each other. The digestibility coefficient for fibre at $5 \%$ inclusion level was significantly high $(\mathrm{P}<0.05)$ for the boiled locust bean diet $(87.90 \%)$ followed by the fermented diet $(32.50 \%)$, the raw diet $(31.89 \%)$ which are not significantly different $(\mathrm{P}>0.05)$ from each other but significantly different $(\mathrm{P}<0.05)$ from the toasted diet (28.40\%).

At $10 \%$ inclusion level, the boiled diet still had a significantly high $(\mathrm{P}<0.05)$ value $(59.60 \%)$ than the fermented $\operatorname{diet}(33.90 \%)$ which is not different from the control diet (31.90\%) but significantly different $(\mathrm{P}<0.05)$ from the toasted diet $(26.80 \%)$ and the raw diet $(21.02 \%)$. Furthermore, with increase in inclusion level to $15 \%$, the boiled diet was still had the best ADC value $(45.90 \%)$ followed by the fermented diet $(37.40 \%)$ which is significantly different from the toasted diet (37.90\%), the control diet $(31.90 \%)$ and the raw diet $(31.10 \%)$. The mineral digestibility was significantly high $(\mathrm{P}<0.05)$ for the toasted diet $(96.03 \%)$ at $5 \%$ inclusion level. The value is higher than those of fermented diet (84.89\%), raw diet (74.30\%), boiled diet $(60.50 \%)$ while the control diet gave a significantly low ADC value of $20.40 \%$.

For $10 \%$ inclusion level, the fermented locust bean diet gave a significantly high value (54.90\%) than toasted diet (49.40\%) which is not significantly different $(\mathrm{P}>0.05)$ from the boiled diet $(40.40 \%)$ but significantly different $(\mathrm{P}<0.05)$ from the raw and the control diets with values $(24.80 \%$ and $20.40 \%$ respectively) 
that are not significantly different from each other $(\mathrm{P}>0.05)$. At 15\% inclusion level, the raw locust bean based diet had a significantly high value $(49.90 \%)$ than the fermented diet $(40.90 \%)$, and the boiled diet (37.70\%) which is not significantly different $(\mathrm{P}>0.05)$ from that of toasted duet $(35.50 \%)$ but significantly different from the control diet (20.40\%).

\section{Discussion}

From the result, Clarias gariepinus responded to all the thirteen (13) diets. The fish growth thus, was positively affected by the processing methods of the locust bean meal as its inclusion levels in the diets. The fermented and toasted locust bean meal based diets expressed their best impacts on the growth of the fish at maximum inclusion levels of $5 \%$ beyond which there were declines in the growth parameters. This could be attributed to the effect of fermentation which could have affected the palatability of the diets. This is in line with the report of Samuel et al. [25], which says that high inclusion of fermented feeds in diet of animal reduces feed intake. Similarly, the decline in the performance of toasted locust bean meal based diet could also be due to the effect of temperature on the seed which might have denatured the protein content and thus, rendering it unavailable, less soluble and less digestible Ironkwe \& Esonu [26], Orire \& Ricketts [27].

Furthermore, the poor nutrient utilization for the fermented diet can also be attributed to high anti-nutritional compounds in the diet which is high in tannin, hydrocynanide, and saponin compared with other treatments. However, Alabi et al. [28], Jauncy [29] who reported that, nutrient utilization should be reflected in the flesh of the fishes carcass compositions. The cooked locust bean meal based diet was utilized up to $15 \%$ inclusion level this is also observed with raw locust bean meal based diet. The raw locust bean meal utilization up to $15 \%$ could be as a result of the body nutrient requirement which needed to be supplied in large quantity in view of the limiting nutrients in the diet. It would be observed that, the raw had the highest ANF values, high fibre content as well as poorest digestibility values which will affect its utilization.

This is in agreement with the work of Fapohunda [10] who reported that high fiber content can limit protein availability or growth. Thus, for the nutrients to be available for growth enough of it is needed to be supplied. Similarly, of all the treatment the diet with poorest growth values was the raw locust bean based diets at all levels of inclusion. This means that majority of the fishes rejected this diet and thus had poor feed conversion rate [25] and that the fishes that continued with this diet utilize available nutrients for growth and body compositions at a high rate $[28,30]$. Meanwhile, irrespective of the processing methods and inclusion levels of the locust bean meal based diets, the control diet ( $0 \%$ locust bean meal) had the best performance in growth response overall. This could be due to the high protein and mineral content of the fish meal in the diet. This is in agreement with the report of Pandey [31], which says that feed intake increase in animals if the aroma of diet is pleasant, which is true of fishmeal.

The utilization could also be attributed to absence of antinutritional factors as well as low fibre content of fish meal which allows highly nutritive minerals such as lysine and methionine to be readily available to the fishes [32-35].

\section{Recommendations}

From the research, it can be recommended that for the inclusion of locust bean meal in the diet of Clarias gariepinus fingerlings, farmers should can substitute fish meal with locust bean meal at $5 \%$ if fermented and toasted and at $15 \%$ if raw and cooked respectively.

\section{References}

1. FAO (2009) Practical Guide to Nutrition, Feeds and Feeding of Catfish.

2. Macel D, Sales J, Janssens PJG (2003) Nutrient requirements of Ornamental fish. Review Aquatic Living Resour 16: 533-540.

3. Anderson JI (2003) International Seafood Trade. Wood Head Publishing Ltd. Cambridge, England.

4. Guttormsen AG (2002) Input Substitutability in Salmon. Aquaculture Marine Resource Economics 17: 91-102.

5. De Silva SS, Hasan MR (2007) Feeds and fertilizers: the key to long term sustainability of Asian aquaculture. In: Hasan MR Hecht T, De Silva SS, Tacon AGJ (Eds.), Study and analysis of feeds and fertilizers for sustainable aquaculture development. pp. 19-47.

6. FAO (2010) Cultured aquatic species information programme. Fisheries and Aquaculture Organization of United Nations, Italy.

7. Oluwole SI, Oluremi OK (2012) Comparison between the Amino Acid, Fatty Acid, Mineral and Nutritional Quality of Raw, Germinated and Fermented African Locust Bean (Parkia biglobosa) Flour. Acta Sci Pol Technol Aliment 11(2): 151-165.

8. Steinkraus KH (1996) Handbook of Indigenous Fermented Foods. ( $2^{\text {nd }}$ edn).

9. Ikenebomeh MJ, Kok R (1984) Mass balance of the processing and fermentation of the African locust bean (Parkia fi licoidea Welw). J Can Inst Food Sci Tech 17: 48-50.

10. Fapohunda 00 (2012) Evaluation of processed soybean meal in the feeding of Clarias gariepinus fingerlings. Journal of animal science Advances 2(2): 244-249.

11. Lind OT (1979) Hand Book of common method in Limnology. ( $\left.2^{\text {nd }} e d n\right)$, The CV Moby publisher, USA, pp. 199.

12. APHA (1980) Standard Methods for the Examination of water and waste water. $\left(18^{\text {th }}\right.$ edn $)$, American Public Health Association, Washington DC, USA, p. 1228.

13. Fakunle JO, Alatise SP, Effiong BN, Tiomiyo K (2013) Effects of replacing soya bean with graded levels of boiled Jatropha Kernel in diets of Clarias gariepinus fingerlings. Bulletin of Environment, Pharmacology and Life Sciences 2(9): 112-117.

14. Yusuf OIS, Raji MAY (2012) The processing and preference for Locust Bean Product in Lagos. Journal of biology, Agriculture and Health care 2(11): 2224-3208.

15. Nwachukwu NC, Wokem GN, Azoro C, Adeleye 00, Ezenlee A (2005) Effects of roasting methods on the nutritive of its protein quality. Yankari Journal 2: 28-32. 
16. Pearson DA (1976) Chemical analysis of foods. ( $7^{\text {th }}$ edn), Churchill living stone, Edinburgh, Scotland, UK, England.

17. Gregory I, Onwuka RA (2005) Food Analysis and Instrumentation Theory and Practice, Naphthals Prints. A Division of H.G. Support Nig. Ltd. Adeniyi Jones Surulere, Nigeria.

18. Maynard LA, Loosli JK, Hintz HF, Waener RG (1979) Animal Nutrition. ( $7^{\text {th }}$ edn) McGraw Hill, New York, USA, pp. 620.

19. Halver JE (1989) Fish Nutrition. ( $2^{\text {nd }}$ edn), Academic Press, New York, USA, pp. 875 .

20. Brown ME (1957) Experimental Studies on Growth. In: Hoar WS, Randell DJ (Eds.), Physiology of Fishes. Academic Press New York, USA, pp. 361-400.

21. Osborne T, Mendel L, Ferry E (1919) A method for expressing Numerically the Growth Promoting Value of Proteins. Journal of Biochemistry 37: 223-229.

22. Bondi AA (1987) Animal Nutrition. John Wiley and Sons, New York, USA, pp. 504.

23. Miller DS, Bender AE (1955) The Determination of the Net Protein Utilization by a Shortened Method. British Journal of Nutrition 9: 382388

24. Cockrel I, Halliday D, Morgan DJ (1987) Studies on the nutrition of marine fish utilization of various dietary proteins by plaice. (Pleuronectes platessa). British Journal of Nutrition 31: 297-306.

25. Samuel O, Akpet UBA, Orok EE, Ayuk EA, Essien AKU (2012) Effect of fermented locust bean seed Parka clapatonaina as a replacement for full fat soya bean meal on the performance and haemathological parameters of weaner rabbits. Journal of biology, aquaculture and health care 2(2): 23-43.
26. Ironkwe MO, Esonu BM (2012) Effect of raw and toasted Bambara groundnut (vigina subterranean (L) verd court on the performance of broiler finisher bird in Global. Journal of Bio-Science and Biotechnology 1: 29-32.

27. Orire AM, Rickets OA (2013) utilization of mellon shell as dietary source in the diet of nice Tilapia (Qreochromis Nicoticus). Research inventory: International Journal of Engineering and Science 2(4): 5-11.

28. Alabi DA, Akindahunsi OR, Sanyaolu MA (2005) Quantitative determination of Chemical and nutritional composition of Pakia biglobosa (Jacq) Benth. African Locust bean Seeds. African journal of Biotechnology 4(8): 812-815.

29. Church D, Pond J (1998) Basic Animal Nutrition and Feeding. ( $3^{\text {rd }}$ edn). pp. 572.

30. Ayanwale BA, Ari MM (2002) Replacement of Parkia filicoidea in Broiler. Journal of Agriculture and Agriculture Technology 1(1): 42-52.

31. Pandey G (2013) Feed formulation and feeding technology for fishes. Int Res J Pharm 4(3): 23-30

32. Suriamsory S, Silman P (2008) Nutritive composition of soya bean byproducts and nutrient digestibility of soya bean pod husk. International Journal of science and technology 2(03): 568-576.

33. AOAC (2007) Official methods of analysis. (17 th $\mathrm{edn})$ Association of Official Analytical Chemist, Gaithersburg, Maryland, USA.

34. FAO Fisheries Technical Paper No. 497. FAO, Rome, Italy, pp. 510.

35. FAO (2005) Globe Fish Commodity Update, Fishmeal land Fish oil. Rome, Italy.

\section{Your next submission with Juniper Publishers will reach you the below assets}

- Quality Editorial service

- Swift Peer Review

- Reprints availability

- E-prints Service

- Manuscript Podcast for convenient understanding

- Global attainment for your research

- Manuscript accessibility in different formats ( Pdf, E-pub, Full Text, Audio)

- Unceasing customer service

Track the below URL for one-step submission https://juniperpublishers.com/online-submission.php 